\title{
Effect of Soy Isoflavones on the Performance of Cerebral Neurotransmitter Systems in Adult and Aged Male Rats
}

\author{
Mohammad A. Alhazmi, Fares K. Khalifa and Mustafa A. Zeyadi \\ Biochemistry Department, Faculty of Science, King Abdul-Aziz University, KSA
}

\section{ABSTRACT}

The aim of this study was to investigate the effects of Soy isoflavone on the performance of neurotransmitter systems in adult and aged male rats. Methods: Twenty adult male Sprague-Dawley rats weighing 183.2- 184.2 g (6-8 months) and twenty aged male albino rats weighing 405.5- $429 \mathrm{~g}$ (12-16 months) divided to four groups (10 rats per group). as follow: G1: Adult control group (Adult-C) ; rats fed on basal diet; G2: Aged control group (Aged-C); rats fed on basal diet; G3: Adult Isoflavone group (Adult-ISO) rats fed on basal diet and received isoflavone (100 mg /day) ;G4: Aged Isoflavone group (Aged-ISO); rats fed on basal diet and received isoflavone (100 mg/day) dissolved in $10 \mathrm{ml}$ of distilled water by oral gavage. At the end of experiment (four weeks) blood samples were collected for biochemical measurements. Result: Current evidence revealed that, soy isoflavone treated group showed enhancement in dopamine, serotonin, norepinephrine, nitric oxide, acetylcholine, glutamate and GABA whereas decrease in choline acetyl transferase. Conclusion: soy isoflavones improved the neurotransmitters and brain cells functionally and lead to improve the cognitive functions and memory.

\section{INTRODUCTION}

The nervous system requires seamless communication between numerous nerve cells to function effectively. Centrally, this necessitates endogenous neurotransmitter (NT) release; NTs attach to dedicated receptors on target neurones, altering their activity. NTs are therefore crucial biochemical compounds that govern behavioural and physiological properties within both central and peripheral nervous organisations (Moon et al., 2018). A NT acts as a chemical messenger that conveys information over a neurological synapse, e.g. neuromuscular junction; signals are transferred from the initial neurone to the

\section{ARTICLE INFORMATION}

Received 29th Oct 2020 Accepted after revision 14th Dec 2020 Print ISSN: 0974-6455 Online ISSN: 2321-4007 CODEN: BBRCBA

Thomson Reuters ISI Web of Science Clarivate Analytics USA and Crossref Indexed Journal

\section{Clarivate
Analytics}

NAAS Journal Score 2020 (4.31)

A Society of Science and Nature Publication,

Bhopal India 2020. All rights reserved.

Online Contents Available at: http//www.bbrc.in/

Doi: http://dx.doi.org/10.21786/bbrc/13.15/11 target. The latter may include another nerve, muscle or gland cell (Lodish et al., 2000).

NTs are produced by synaptic vesicles within the nerve axon terminals. They are discharged into the synaptic cleft and act on the relevant target cell receptors. The chemicals are manufactured from straightforward and numerous amino acid building blocks. The latter are easily accessed from food intake and require minimal modification for NT synthesis. The resulting NTs are key components in the facilitation of normal daily activities (Steven, 2005). They are also principle actors in several neurodegenerative pathologies, including Alzheimer's dementia, Parkinson's disease and epilepsy. Not all NTs have yet been fully characterised; the inventory is constantly expanding as further compounds are discovered (Chauhan et al., 2020).

In Asia, soy has been a customary part of the diet for many years. However, in western nations, it is much less popular, despite that fact that soy is a highly proteinaceous, fibrous food that also contains a range of phytochemicals. Henry-Vitac et al. (2010) have suggested soy and isoflavone consumption offer a number of

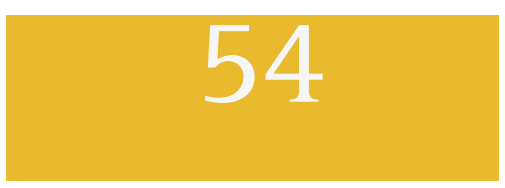


advantages to well-being. The many phytochemicals present in soy include isoflavones, phytic acid, trypsin inhibitors and saponins (Anderson and Wolf, 1995). In particular, the beneficial properties of isoflavones have been the subject of interest (File et al., 2001).

A plethora of research has been performed to evaluate the possible positive impact of soy isoflavones on a range of pathologies associated with ageing. However, few studies have evaluated their effect on cognitive abilities. Different forms of nerve injury, including those arising from advancing years, may give rise to neurodegenerative conditions that are associated with impaired cognitive function. It has been shown that cognitive function can be enhanced in both humans and rats by soy isoflavones (Pan et al., 2000) although the exact mechanism of this effect has not yet been elucidated. Soy isoflavones have been demonstrated to exhibit oestrogenic properties; imitation of oestrogen within the central nervous system may underlie some of these observations (Pan and Clarkson, 1999), but may not represent the whole story.

\section{MATERIAL AND METHODS}

Soy isoflavone were obtained as natural product as tablets, each serving contains $100 \mathrm{mg}$ of isoflavones (Daidzein $35 \mathrm{mg}$, Puerarin $33 \mathrm{mg}$, Genistein $27 \mathrm{mg}$, Glycitein $5 \mathrm{mg}$ )

Chemicals: Kits of Dopamine, Serotonin, Glutamate, Norepinephrine, Gamma-aminobutyric Acid, Choline Acetyltransferase, Acetylcholine, and Nitric Oxide were obtained from Bioassay Technology Laboratory (Shanghai, China).

Experimental Animals: Twenty adult male albino rats weighing 183.2-184.2 g (6-8 months) and twenty aged male albino rats weighing 405.5- $429 \mathrm{~g}$ (12-16 months) will be used in this study. Rats will be supplied from the Animal House Colony of King Fahd Medical Research
Centre, Jeddah. After the acclimatization period, Rats will be controlled with a $12 \mathrm{~h}$ light/dark cycle at King Fahd Medical Research Centre Animal Facility Breeding Colony. Rats will be housed with adlibitum access of water and standard laboratory diet. Experiment will be approved by the Ethical Committee of King Fahd Medical Research Centre. Jeddah, KSA.

Experimental Design: Forty male rats were randomly divided to four groups (10 rats per group). The groups were treated as follows:

GROUP I: Adult control group (Adult-C) ; rats fed on basal diet

GROUP II: Aged control group (Aged-C); rats fed on basal diet

Group III: Adult Isoflavone group (Adult-ISO) rats fed on basal diet and received isoflavone (100 $\mathrm{mg} /$ day) dissolved in $10 \mathrm{ml}$ of distilled water by oral gavage.

GROUP IV: Aged Isoflavone group (Aged-ISO); rats fed on basal diet and received isoflavone (100 $\mathrm{mg} /$ day) dissolved in $10 \mathrm{ml}$ of distilled water by oral gavage.

Sample Collection: At the end of four weeks (experimental period). The animals were starved for 12 hours prior to blood collection. Rats were sacrificed under ether anesthesia, then blood was collected through optic nerve puncture. Serum was separated by allowing blood samples left for 30 minutes at temperature of $25^{\circ} \mathrm{C}$ then centrifuged at 5000 r.p.m for 10 minutes, then divided into several aliquots and stored at -20C until analysis was performed.

Statistical Analysis: Data were statistically analyzed by comparing the values for different experimental groups with the values of individual normal ones. Results were expressed as mean + S.E. Significant differences among groups were analyzed using analysis of variance ONEWAY ANOVA coupled with Statistical Package for the Social Science (SPSS) program. ANOVA at $p<0.05$ was considered significant.

\begin{tabular}{|c|c|c|c|c|}
\hline \multirow{2}{*}{ Parameter } & \multicolumn{2}{|c|}{ Control (C) } & \multicolumn{2}{|c|}{ Isoflavone (ISO) } \\
\hline & Adult G1)) & Aged(G2) & Adult(G3) & Aged (G4) \\
\hline $\begin{array}{l}\text { Dopamine } \\
\text { (ng/mL) }\end{array}$ & $1.53 \pm 0.95^{\mathrm{ac}}$ & $1.21 \pm 0.14^{\mathrm{bc}}$ & $3.06 \pm 0.24^{\mathrm{ad}}$ & $2.39 \pm 0.23^{\text {bd }}$ \\
\hline $\begin{array}{l}\text { Norepinephrine } \\
\text { (ng/L) }\end{array}$ & $207.4 \pm 8.2 \mathrm{a}$ & $169.5 \pm 12.1^{\mathrm{abc}}$ & $208.3 \pm 10.8^{b}$ & $199.7 \pm 13.8^{c}$ \\
\hline
\end{tabular}

\section{RESULTS}

Biochemical Findings: Table1 shows the effects of isoflavone on serum levels of biogenic catecholamine neurotransmitters dopamine and norepinephrine of experimental rats. Lower dopamine levels were recorded in aged control rats (Aged-C) when compared to adult control rats (Adult-C). On the other hand, the results of the present study showed significant $(p \leq 0.05)$ higher levels of serum dopamine in adult isoflavone rats (Adult- 
Iso) as compared with adult control one. An improvement in the levels of serum dopamine levels were observed in aged isoflavone treated rats (Aged-Iso) when compared to aged isoflavone group. No significant differences in serum norepinephrine levels were found among adult control, adult isoflavone, and aged isoflavone treated rats. Lower epinephrine levels were recorded in aged control group when compared to all other groups. Consumption of isoflavone has significant improvement $(\mathrm{P} \leq 0.05)$ on serum epinephrine in aged rats (Aged-Iso) as compared to aged control (Aged-C).

Table 2 shows the effects of isoflavone on serum levels of excitatory neurotransmitters glutamate (Glu) and acetyl choline (Ach) of experimental rats. No significant differences in serum Glu levels were found among adult control, adult isoflavone, and aged isoflavone treated rats. Lower Glu levels were recorded in aged control group when compared to all other groups. Consumption of isoflavone has significant improvement $(\mathrm{P} \leq 0.05)$ on serum Glu in aged rats (Aged-Iso) as compared to aged control (Aged-C). Lower Ach levels were recorded in aged control rats (Aged-C) when compared to adult control rats (Adult-C). The results of the present study showed significant increase $(\mathrm{p} \leq 0.05)$ in Ach serum levels in adult isoflavone rats (Adult-Iso) as compared to adult control one. Supplementation of isoflavone resulted in significant increase in the levels of serum excitatory neurotransmitters acetylcholine in both adult and aged groups (Adult-Iso and Aged-Iso) when compared to control groups (Adult-C and Aged-C) respectively.

\begin{tabular}{|c|c|c|c|c|}
\hline \multirow{2}{*}{$\begin{array}{l}\text { Table 2. Ef } \\
\text { Neurotransn } \\
\text { Parameter }\end{array}$} & \multicolumn{2}{|c|}{ Control (C) } & \multicolumn{2}{|c|}{ Isoflavone (ISO) } \\
\hline & Adult G1)) & Aged(G2) & Adult(G3) & Aged (G4) \\
\hline $\begin{array}{l}\text { Glutamate } \\
(\mu \mathrm{g} / \mathrm{mL})\end{array}$ & $16.7 \pm 2.2 \mathrm{a}$ & $12.8 \pm 1.6 a b c$ & $17.5 \pm 2.0 \mathrm{~b}$ & $17.8 \pm 5.1 \mathrm{c}$ \\
\hline $\begin{array}{l}\text { Acetyl Choline } \\
(\mu \mathrm{g} / \mathrm{L})\end{array}$ & $58.3 \pm 7.48 \mathrm{ac}$ & $41.8 \pm 5.1 b c$ & $67.8 \pm 4.9 \mathrm{ad}$ & $55.7 \pm 5.3 \mathrm{bd}$ \\
\hline \multicolumn{5}{|c|}{$\begin{array}{l}\text { Results were represented as means } \pm S D, n=10 \text {, means bearing the same } \\
\text { alphabets in the same row are significantly different at }(\mathrm{p} \leq 0.05)\end{array}$} \\
\hline \multicolumn{5}{|c|}{$\begin{array}{l}\text { Table 3. Effect of different treatments on serum levels of Inhibitory } \\
\text { Neurotransmitter Gamma Amino Butyric Acid (GABA) and Serotonin }\end{array}$} \\
\hline \multirow[t]{2}{*}{ Parameter } & \multicolumn{2}{|c|}{ Control (C) } & \multicolumn{2}{|c|}{ Isoflavone (ISO) } \\
\hline & Adult G1)) & Aged(G2) & Adult(G3) & Aged (G4) \\
\hline $\begin{array}{l}\text { Gama Amino } \\
\text { Butyric Acid } \\
\text { (nmol/L) }\end{array}$ & $16.7 \pm 2.2 \mathrm{a}$ & $12.8 \pm 1.6 \mathrm{abc}$ & $17.5 \pm 2.0 \mathrm{~b}$ & $17.8 \pm 5.1 \mathrm{c}$ \\
\hline $\begin{array}{l}\text { Serotonin } \\
(\mathrm{ng} / \mathrm{mL})\end{array}$ & $58.3 \pm 7.48 \mathrm{ac}$ & $41.8 \pm 5.1 \mathrm{bc}$ & $67.8 \pm 4.9 \mathrm{ad}$ & $55.7 \pm 5.3 \mathrm{bd}$ \\
\hline
\end{tabular}

Table 3 shows the effects of isoflavone on serum levels of inhibitory neurotransmitter gamma amino butyric acid (GABA) and serotonin of experimental rats. Lower GABA and serotonin levels were recorded in aged control group when compared to all other groups. Consumption of isoflavone has significant improvement $(\mathrm{P} \leq 0.05)$ on serum GABA levels in aged rats (Aged-Iso) as compared to aged control (Aged-C).

No significant differences in serum choline acetyl transferase (ChAT) activity were found among adult control, adult isoflavone, and aged isoflavone treated rats. Lower ChAT levels were recorded in aged control group when compared to all other groups (Figure 1). Consumption of isoflavone has significant improvement ( $\mathrm{P} \leq 0.05)$ on serum ChAT in aged rats (Aged-Iso) as compared to aged control (Aged-C). Lower NO levels were recorded in aged control rats (Aged-C) when compared to adult control rats (Figure 2). Supplementation of isoflavone resulted in significant increase in the values of serum nitric oxide in aged rats (Aged-Iso) as compared to aged control rats. 
Figure 1: Effect of different treatments on serum level of Choline Acetyl Transferase $(\mu \mathrm{g} / \mathrm{mL})$ in experimental rat groups. Adult-C: Control adult rat group; Aged-C: Control aged rat group; Adult-Iso: Adult rats received Isoflavone (100mg/ day); Aged-Iso: Aged rats received Isoflavone (100mg/ day).

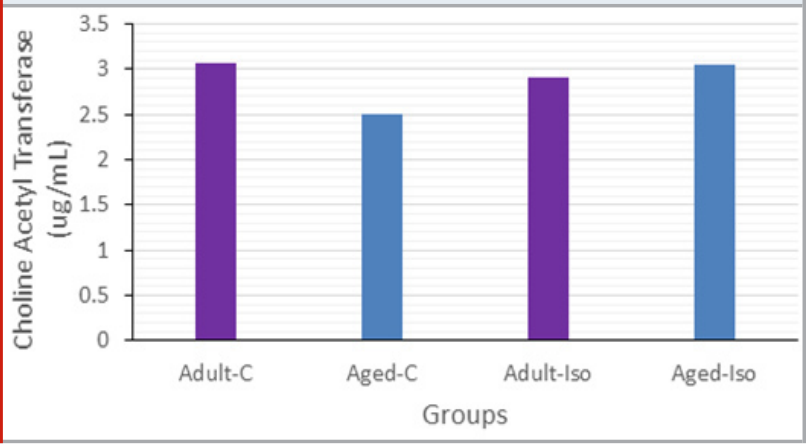

Figure 2: Effect of different treatments on serum level of Nitric Oxide $(\mu \mathrm{mol} / \mathrm{mL})$ in experimental rat groups. Adult-C: Control adult rat group; Aged-C: Control aged rat group; Adult-Iso: Adult rats received Isoflavone (100mg/ day); Aged-Iso: Aged rats received Isoflavone (100mg/ day).

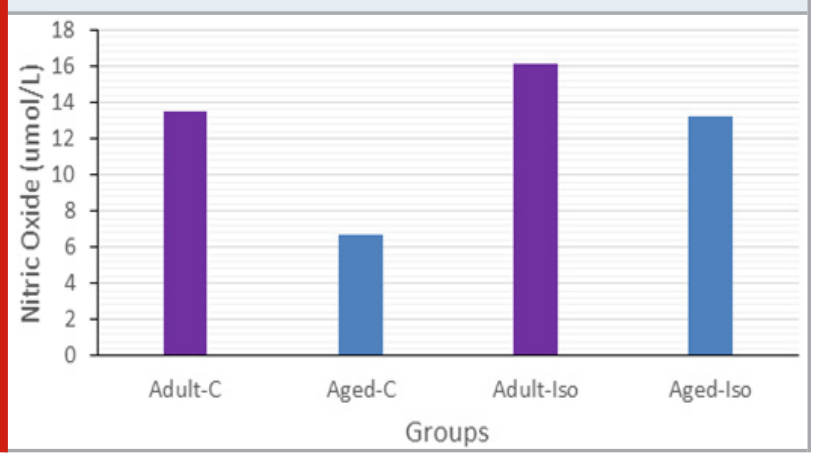

\section{DISCUSSION}

The current research has shown that soy isoflavones impact the efficiency of NT systems in both adult and aged male rats. Alterations in cerebellar noradrenergic and serotonergic neurotransmission may have grave repercussions for the normal performance of the entire nervous system. Additionally, collaboration between intra-cerebral territories may be impaired. Serotonin (5-hydroxytryptamine (5-HT)) is known to influence mood; both stress and steroids have an impact on its neurotransmission (Bethea et al., 2000). This NT also acts as a presynaptic autoreceptor. It exhibits a forceful regulatory influence over serotonin neurone firing rates and consequently, on serotonin outflow within the forebrain (Lanfumey and Hamon, 2000).

Numerous 5-HT1A receptors are expressed on postsynaptic membranes throughout the brain, but they are particularly concentrated within the limbic system, notably the hippocampus, and cerebral cortex (Varnäs et al., 2004). Serotonin influences the various growth stages of the cerebellum. It governs dendritic development, together with the creation and balancing of synaptic junctions (Suwa et al., 2014). The results from this work demontrated enhanced serotonin levels in the adult treated group compared with the adult control cohort, i.e. $20.6 \mathrm{ng} / \mathrm{mL}$ and $13.7 \mathrm{ng} / \mathrm{mL}$, respectively. In the aged group, a smaller rise was noted compared with the aged control group, i.e. $14.2 \mathrm{ng} / \mathrm{mL}$ compared with 9.1 $\mathrm{ng} / \mathrm{mL}$, respectively. Some researchers have proposed that augmented serotonin titres, e.g. through ingesting soy isoflavones, are related to a reduction in forebrain serotonin metabolism (Saied and Hassan, 2014).

In addition, cerebellar aberrations of serotonin homeostasis may predipsose to symptomatology characteristic of some psychiatric pathologies (Ivanov et al., 2014). The NTs liberated at individual synapses largely dictates their role; the essential functional dichotomy is excitatory versus inhibitory synapses. Gamma-aminobutyric acid (GABA) is the priniciple inhibitory NT that participates in the majority of cerebral neuronal coding and processing. Its activity is mediated through ionic GABAA receptors and G-protein coupled GABAB receptors. Membrane potentials are impacted via the former; the latter governs synaptic and whole network flexibility over short and long periods of time (Inoue et al., 2013). In the current study, data indicated that GABA concentrations were diminished in the aged control rats compared with the adult control group. In contrast, GABA titres in the aged rats that received soy isoflavones were elevated compared with the aged controls. Therapy with soya isoflavones achieved an equilibrium between both inhibitory, i.e. serotonin and GABA, and excitatory, i.e. glutamate, NTs, thus offering a neuroprotective action (Bañuelos et al., 2014).

Glutamate is composed of several endogenous amino acids; learning and memory are strongly associated with its excitatory NT properties (Meldrum, 2000). No changes were seen in either of the two adult rat cohorts, i.e. Adult-C and Adult-Iso, or in the aged group that had isoflavone. However, the aged control group exhibited a diminished glutamate concentration in comparison. Thus soya isoflavone therapy enhances glutamate titres in older rats; these results are related to improved cognitive function (Sandini et al., 2019). This study suggests that ingestion of isoflavone markedly affects neurotransmission in the cerebellum, particularly in relation to the noradrenergic and serotonergic components. Serum epinephrine levels were enhanced following isoflavone administration in the elderly rats (Aged-Iso) compared to their controls (Aged-C). Norepinephrine (NE) titres were also elevated in the Aged-Iso cohort; this may indicate an unwanted side issue relating to the effect of these chemicals on emotions and life behaviour (Matusda et al., 2010).

NE functions as a central and peripheral NT, and as a hormone. It is associated with the 'fight or flight' reflex that occurs in response to potential threat; its effects are facilitated by a group of adrenoceptors (Bylund, 
2016). One of its consequences is elevation of both systolic and diastolic systemic pressures. Dementia is a neurodegenerative pathology that is associated with gradual cognitive impairment. It is typically seen in adults over the age of 65 years; the prevalence of dementia is significant worldwide. The NT dopamine (DA) is a key component of the central reward pathway and in the control of movement. In the former, DA is manufactured within nerve cell bodies in the ventral tegmental territory, from where it is exuded into the nucleus accumbens and prefrontal cortex (Juárez et al., 2016). DA contributes to many cerebral functions including behaviour and cognition, motivation, sleep, dreaming, mood, attention, working memory and learning (Juárez et al., 2016).

In this study, DA concentrations were diminished in the aged control cohort compared with the adult control group. However, in rats treated with isoflavones, a marked rise in DA titres were noted in the adults compared to the aged group. Enhanced serum DA levels were also measured in the aged isoflavone-treated rats (Aged-Iso) as opposed to in the aged isoflavone group. It was presumed that the rise in DA may either potentiate its manufacture or diminish its breakdown; this was confirmed since isoflavone administration caused a decrease in monoamine oxidase levels. Given the oestrogenic properties of soya isoflavone, ingestion of genistein, a natural isoflavone phytoestrogen that occurs in soybeans, may lead to enhancement of amphetaminetriggered striatal DA release in rats of both sexes through an action equivalent to that of oestradiol (Ferguson et al., 2002).

Acetylcholine (ACh) is thought to be one of the most longstanding central and peripheral NTs that has been identified in animals. In the peripheral nervous system, ACh acts on designated receptors to control muscle function. Within the central nervous system, Ach contributes to the control of various behaviours. ACh is produced within neurones from its precursor, choline, a process mediated via choline acetyltransferase (ChAT) and acetyl coenzyme A (Strandwitz, 2018).

Results from the current study demonstrated that ACh titres in the adult treated group were elevated compared with those in the adult control group, i.e. $67.8 \mathrm{ug} / \mathrm{mL}$ and $58.3 \mathrm{ug} / \mathrm{mL}$, respectively. Similar results were found in the aged rats, with the levels detected in the treated and control groups measured at $55.7 \mathrm{ug} / \mathrm{mL}$ and 41.8 $\mathrm{ug} / \mathrm{mL}$, respectively. It is recognized that soy isoflavone impacts the central cholinergic system, and consequently cognition, by inhibiting acetylcholinesterase (AChE) and thus diminishing ACh concentration (Yang et al., 2011).

Daidzein, an isoflavone, may play an essential part in ACh synthesis in the form of a ChAT activator (Heo et al., 2006). ChAT is crucial for ACh synthesis; it catalyses the acetyl group transfer to choline from acetyl-CoA. One of the findings noted in Alzheimer's disease is that there is a reduction in ChAT expression and function
(Kumar et al., 2107). It has been postulated that ChAT could act as a biomarker for the early recognition of this neurodegenerative condition and other types of dementia. Isoflavone gradients can bind to ChAT specifically; this may assist in screening the well-being of cholinergic neurones, thus offering a vital instrument to predict early outcomes in Alzheimer's disease (Kumar et al., 2017).

In the current research, isoflavone ingestion led to augmented serum ChAT titres in the aged rats (AgedIso) as opposed to their age-equivalent controls. Soy isoflavones have been identified both in vitro and in vivo as free radical quenching agents (Rimbach et al., 2003). These observations have led to the theory that isoflavones' antioxidant properties can impact the stability of compounds such as nitrous oxide (NO), an endothelium-derived vasoactive agent. This may trigger a fall in blood pressure, since NO has been described as a vasodilating NT (Okamura et al., 2002).

Furthermore, NT also functions as a synaptic retrograde NT, which regulates cerebral circulation and additionally contributes to intraneuronal signalling, encompassing neuronal metabolic pathways and development of dendritic spines (Picón-Pagès et al., 2019). Excitation of neurones leads to increased intracellular calcium; this occurs via N-type Ca2+ channels. Neuronal NO synthase is then triggered to manufacture NO; NO then initiates vascular dilatation mediated through a cyclic GMP-dependent pathway. Basal NO activity has been demonstrated to rise following brief soy isoflavone administration (Sobey et al., 2004). In this work, when contrasted with their respective control cohorts, both the adult and aged rats that received isoflavones exhibited elevated NO concentrations for four weeks.

In view of the above, the hypotensive actions of soy powder noted in this study may be associated with NOpotentiated vasodilatation. Marked elevation of serum NO titres were seen following isoflavone administration. These findings are in keeping with the results of Sobey et al. (2004). These authors showed that in male rats, a brief course of daidzen influenced cerebral arterial reactivity; this effect was a consequence of increased manufacture and release of NO, originating from vascular endothelium. Squadrito et al. (2002) reported that flowmediated endothelium-dependent vasodilatation was augmented in normal postmenopausal females following six months of genistein administration. The proportion of serum NO to endothelin, a vasoconstricting agent, was elevated. The vasodilating effect of NO safeguards against the onset of hypertension and concomitant cardiovascular disease (Taddei et al., 2000). Oxidative stress is related to diminished NO levels; the latter is also linked with elevated superoxide anion formation. This can ameliorate the beneficial effects of NO, thus aggravating hypertension in humans and in experimental scenarios. It can be presumed that a rise in serum NO could be facilitated by the antioxidant influence of isoflavone in the aged isoflavone-treated cohort. 


\section{REFERENCES}

Anderson, R. L., \&t Wolf, W. J. (1995). Compositional changes in trypsin inhibitors, phytic acid, saponins and isoflavones related to soybean processing. The Journal of nutrition, 125(suppl_3), 581S-588S.

Bañuelos, C., Beas, B. S., McQuail, J. A., Gilbert, R. J., Frazier, C. J., Setlow, B., \&t Bizon, J. L. (2014). Prefrontal cortical GABAergic dysfunction contributes to age-related working memory impairment. Journal of Neuroscience, 34(10), 3457-3466.

Bethea, C. L., Mirkes, S. J., Shively, C. A., \&t Adams, M. R. (2000). Steroid regulation of tryptophan hydroxylase protein in the dorsal raphe of macaques. Biological psychiatry, 47(6), 562-576.

Bylund, D. B. (2016). Norepinephrine. In Reference Module in Biomedical Sciences: Elsevier.

Chauhan, N., Soni, S., Agrawal, P., Balhara, Y. P. S., \&t Jain, U. (2020). Recent advancement in nanosensors for neurotransmitters detection: Present and future perspective. Process Biochemistry, 91, 241-259.

Ferguson, S. A., Flynn, K. M., Delclos, K. B., Newbold, R. R., \&t Gough, B. J. (2002). Effects of lifelong dietary exposure to genistein or nonylphenol on amphetaminestimulated striatal dopamine release in male and female rats. Neurotoxicology and teratology, 24(1), 37-45.

File, S. E., Jarrett, N., Fluck, E., Duffy, R., Casey, K., \& Wiseman, H. (2001). Eating soya improves human memory. Psychopharmacology, 157(4), 430-436.

Henry-Vitrac, C., Berbille, H., Mérillon, J.-M., \& Vitrac, X. (2010). Soy isoflavones as potential inhibitors of Alzheimer $ß$-amyloid fibril aggregation in vitro. Food Research International, 43(8), 2176-2178.

Heo, H. J., Suh, Y.-M., Kim, M.-J., Choi, S.-J., Mun, N. S., Kim, H.-K., . . . Kim, Y. J. (2006). Daidzein activates choline acetyltransferase from MC-IXC cells and improves drug-induced amnesia. Bioscience, biotechnology, and biochemistry, 70(1), 107-111.

Inoue, W., Baimoukhametova, D. V., Füzesi, T., Cusulin, J. I. W., Koblinger, K., Whelan, P. J., . . Bains, J. S. (2013). Noradrenaline is a stress-associated metaplastic signal at GABA synapses. Nature neuroscience, 16(5), 605-612.

Ivanov I, Murrough JW, Bansal R, Hao X, Peterson BS.(2014) Cerebellar morphology and the effects of stimulant medications in youths with attention deficithyperactivity disorder. Neuropsychopharmacology; 39:718-26.

Juárez Olguín, H., Calderón Guzmán, D., Hernández García, E., \&t Barragán Mejía, G. (2016). The Role of Dopamine and Its Dysfunction as a Consequence of Oxidative Stress. Oxidative medicine and cellular longevity, 2016, 9730467.

Kumar, R., Kumar, A., Långström, B., \&t Darreh-Shori, T. (2017). Discovery of novel choline acetyltransferase inhibitors using structure-based virtual screening. Scientific Reports, 7(1), 1-17.

Lanfumey, L., \&t Hamon, M. (2000). Central 5-HT1A receptors: regional distribution and functional characteristics. Nuclear medicine and biology, 27(5), 429-435.

Lodish, H., Berk, A., Zipursky, S. L., Matsudaira, P., Baltimore, D., \&t Darnell, J. (2000). Neurotransmitters, synapses, and impulse transmission. In Molecular Cell Biology. 4th edition: WH Freeman.

Matsuda, S., Saika, S., Amano, K., Shimizu, E., \&t Sajiki, J. (2010). Changes in brain monoamine levels in neonatal rats exposed to bisphenol A at low doses. Chemosphere, 78(7), 894-906.

Meldrum, B. S. (2000). Glutamate as a neurotransmitter in the brain: review of physiology and pathology. J Nutr, 130(4S Suppl), 1007s-1015s.

Moon, J.-M., Thapliyal, N., Hussain, K. K., Goyal, R. N., \&t Shim, Y.-B. (2018). Conducting polymer-based electrochemical biosensors for neurotransmitters: A review. Biosensors and Bioelectronics, 102, 540-552. Okamura, T., Ayajiki, K., Fujioka, H., Shinozaki, K., Et Toda, N. (2002). Pharmacology and Physiology of Perivascular Nerves Regulating Vascular Function: Neurogenic Cerebral Vasodilation Mediated by Nitric Oxide. Japanese journal of pharmacology, 88(1), 3238.

Pan, Y., Anthony, M., \& Clarkson, T. B. (1999). Evidence for up-regulation of brain-derived neurotrophic factor mRNA by soy phytoestrogens in the frontal cortex of retired breeder female rats. Neuroscience letters, 261(1-2), 17-20.

Pan, Y., Anthony, M., Watson, S., \&t Clarkson, T. B. (2000). Soy phytoestrogens improve radial arm maze performance in ovariectomized retired breeder rats and do not attenuate benefits of 17beta-estradiol treatment. Menopause (New York, NY), 7(4), 230-235.

Picón-Pagès, P., Garcia-Buendia, J., \&t Muñoz, F. J. (2019). Functions and dysfunctions of nitric oxide in brain. Biochimica et Biophysica Acta (BBA) - Molecular Basis of Disease, 1865(8), 1949-1967

Rimbach G, De Pascual-Teresa S, Ewins BA, Matsug S, Uchida Y, Minihane AM, Turner R, VafeiAdou K, Wein berg PD. 2003. Antioxidant and free radical scavenging activity of isoflavone metabolites. Xenobiotica 33: 913925.

Saied, N. M., \&t Hassan, W. A. (2014). Long-term exposure to xenoestrogens alters some brain monoamines and both serum thyroid hormones and cortisol levels in adult male rats. The Journal of Basic \& Applied Zoology, 67(5), 205-211.

Sandini, T. M., Reis-Silva, T. M., Moreira, N., Bernardi, M. M., Lebrun, I., \&t Spinosa, H. d. S. (2019). Effects of isoflavones on behavior, estradiol, glutamate, and GABA levels in intact middle-aged female rats. Nutritional Neuroscience, 22(11), 805-816.

Sobey, C. G., Weiler, J. M., Boujaoude, M., \& Woodman, O. L. (2004). Effect of short-term phytoestrogen treatment in male rats on nitric oxide-mediated responses of carotid and cerebral arteries: comparison 
with 17ß-estradiol. Journal of Pharmacology and Experimental Therapeutics, 310(1), 135-140.

Squadrito F, Altavilla D, Morabito N, Crisafulli A, D’Anna R, Corrado F, Ruggeri P Campo GM, Calapai G, Caputi AP, Squadrito G. (2002) The effect of the phy toestrogen genistein on plasma nitric oxide concentra tions, endothelin-1 levels and endothelium dependent vasodilation in postmenopausal women. Atherosclerosis 163: 339-347.

Strandwitz, P. (2018). Neurotransmitter modulation by the gut microbiota. Brain research, 1693, 128-133.

Suwa B, Bock N, Preusse S, Rothenberger A, Manzke T (2017). Distribution of serotonin 4(a) receptors in the juvenile rat brain and spinal cord. J Chem Neuroanat 55:67-77

Taddei S, Virdis A, Ghiadoni L, Salvetti G, Salvetti A (2000). Endothelial dysfunction in hypertension. J Neph rol 13: 205-210.

Varnäs, K., Halldin, C., \&t Hall, H. (2004). Autoradiographic distribution of serotonin transporters and receptor subtypes in human brain. Human brain mapping, 22(3), 246-260.

Yang, H., Jin, G., Ren, D., Luo, S., \&t Zhou, T. (2011). Mechanism of isoflavone aglycone's effect on cognitive performance of senescence-accelerated mice. Brain and cognition, 76(1), 206-210. 